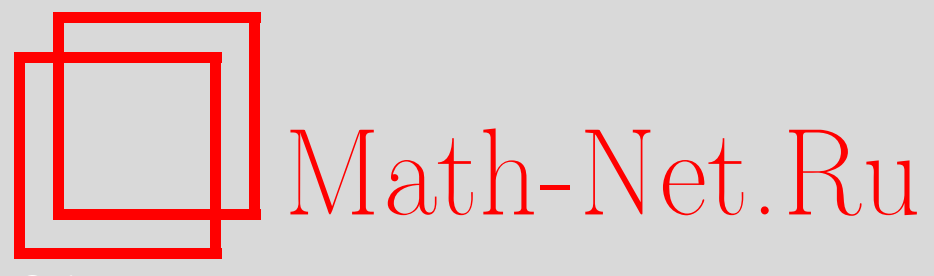

Р. Де Лео, И. А. Дынников, Пример фрактального множества направлений плоскостей, дающих хаотическое пересечение с фиксированной 3-периодической поверхностью, УМН, 2007, том 62, выпуск 5, 151-152

DOI: https://doi.org/10.4213/rm8149

Использование Общероссийского математического портала Math-Net.Ru подразумевает, что вы прочитали и согласны с пользовательским соглашением http://www . mathnet.ru/rus/agreement

Параметры загрузки:

IP: 18.208 .226 .222

26 апреля 2023 г., 14:14:19

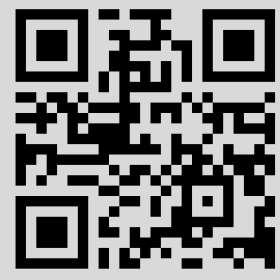




\title{
Пример фрактального множества направлений плоскостей, дающих хаотическое пересечение с фиксированной 3-периодической поверхностью
}

\author{
Р. Де Лео, И. А. Дынников
}

Задача об асимптотическом поведении неограниченных связных компонент плоских сечений 3-периодической поверхности в $\mathbb{R}^{3}$ и строении ассоциированных с ними слоений на поверхности в трехмерном торе $\mathbb{T}^{3}=\mathbb{R}^{3} / \mathbb{Z}^{3}$ была поставлена С. П. Новиковым в [1]. Эта задача происходит из теории нормальных металлов и состоит в следующем. В пространстве $\mathbb{R}^{3}$ с координатами $\left(x^{1}, x^{2}, x^{3}\right)$ фиксирована вложенная поверхность $M$, инвариантная относительно сдвигов на векторы из $\mathbb{Z}^{3} \subset \mathbb{R}^{3}$, гомологический класс проекции которой в торе $\mathbb{T}^{3}=\mathbb{R}^{3} / \mathbb{Z}^{3}$ нулевой. Для всевозможных ковекторов $H=\left(H_{1}, H_{2}, H_{3}\right)$ рассматриваются сечения поверхности $M$ плоскостями $\langle H, x\rangle=$ const (мы будем называть их $H$-сечениями) и ставится вопрос об асимптотическом поведении их неограниченных регулярных связных компонент (если такие имеются). Данный вопрос исследовался в ряде работ [2]-[4]. Физические следствия из полученных результатов обсуждаются в [5].

Возможны следующие три принципиально различных случая поведения $H$-сечений, причем тип сечения одинаков для параллельных плоскостей.

Тривиальный случай. Все компоненты сечения ограничены.

Интегрируемый случай. Каждая регулярная незамкнутая компонента $H$-сечения представляет собой прямую линию, возмущенную конечной деформацией: $\gamma(t)=$ $t \cdot v+O(1), v \in \mathbb{R}^{3} \backslash\{0\}$. Кроме того, имеется ковектор $L_{M, H}$, определенный с точностью до знака, с целыми взаимно простыми координатами $\left(L_{1}, L_{2}, L_{3}\right)$, аннулирующий вектор $v$. Проекция такой компоненты в $\mathbb{T}^{3}$ содержится во вложенном двумерном тоpe, гомологический класс которого двойственен по Пуанкаре ковектору $L_{M, H}$. Мы будем обозначать проективный класс $\left(L_{1}: L_{2}: L_{3}\right) \in \mathbb{R P}^{2}$ ковектора $L_{M, H}$ через $\ell_{M, H}$.

Хаотический случай. Замыкание проекции любой неограниченной компоненты в $\mathbb{T}^{3}$ представляет собой поверхность рода больше двух. Поведение таких компонент не изучено. В некоторых явно построенных примерах такая кривая "блуждает по всей плоскости", т. е. $d$-окрестность кривой есть вся плоскость для некоторого конечного $d$. По-видимому, такое поведение типично для хаотического случая, но это не доказано.

Если $H_{1}, H_{2}, H_{3}$ линейно зависимы над $\mathbb{Q}$, в определениях выше требуются уточнения. Кроме того, в этом случае ковектор $L_{M, H}$ может быть определен неоднозначно. Мы опускаем здесь эти подробности.

Для фиксированной поверхности $M$ и рациональной точки $\ell \in \mathbb{Q P}^{2} \subset \mathbb{R} \mathrm{P}^{2}$ обозначим через $\mathscr{D}_{M, \ell}$ множество $\mathscr{D}_{M, \ell}=\left\{\left(H_{1}: H_{2}: H_{3}\right) \in \mathbb{R P}^{2} ; \ell_{M, H}=\ell\right\}$. Известно, что для 3 -периодической поверхности $M$ общего положения множества $\mathscr{D}_{M, \ell}$ представляют собой не пересекающиеся друг с другом замкнутые области с кусочно гладкой границей, а множество направлений $H$, для которых $H$-сечения хаотичны, имеет меру нуль. Множество направлений $H$, дающих тривиальные $H$-сечения, открыто. Мы называем непустые области $\mathscr{D}_{M, \ell}$ зонами устойчивости.

Для исследования зон устойчивости полезно рассмотреть однопараметрическое семейство $M_{c}=\left\{x \in \mathbb{R}^{3} ; f(x)=c\right\}$ поверхностей уровня фиксированной гладкой функции и ввести обобщенные зоны устойчивости $\mathcal{D}_{f, \ell}=\bigcup_{c} \mathcal{D}_{M_{c}, \ell}$. Они также представляют собой области с кусочно гладкой границей. При $\ell \neq \ell^{\prime}$ зоны $\mathcal{D}_{f, \ell}$ и $\mathcal{D}_{f, \ell^{\prime}}$ могут пересекаться только по граничным точкам, причем множество этих точек не более чем счетно (в известных нам примерах две зоны всегда имеют не более одной общей точки). Если есть хотя бы две обобщенных зоны устойчивости, то тогда этих зон бесконечно много. Для каждой точки дополнения $\left(H_{1}: H_{2}: H_{3}\right) \in \mathcal{E}(f)=\mathbb{R} P^{2} \backslash \bigcup_{\ell} \mathcal{D}_{f, \ell}$ ровно на одном уровне $c$ на поверхности $M_{c}$ имеет место хаотический режим, а на остальных уровнях $H$-сечения тривиальны. Объединение $\bigcup_{\ell} \operatorname{int}\left(\mathcal{D}_{f, \ell}\right)$ представляет

Второй автор поддержан грантом НШ-4182.2006.1. 
собой открытое всюду плотное подмножество в $\mathbb{R P}^{2}$, а его дополнение $\overline{\mathcal{E}(f)}$ имеет вид “двумерного канторовского множества". Однако имеет ли $\mathcal{E}(f)$ нулевую меру для функции $f$ общего положения, до сих пор неизвестно. Согласно гипотезе С. П. Новикова, это множество имеет хаусдорфову размерность строго между 1 и 2.

Указанные выше факты верны для гладких и кусочно линейных поверхностей общего положения. Ряд примеров был исследован численно [6]. В данной работе мы рассмотрим одну кусочно линейную поверхность и опишем для нее зоны устойчивости. Эта поверхность - изогональный изоэдральный полиэдр $\{4,6 \mid 4\}$ из [7]. Ее можно задать уравнением $f=0$, где $f\left(x^{1}, x^{2}, x^{3}\right)=\operatorname{mid}\left(\cos \left(2 \pi x^{1}\right), \cos \left(2 \pi x^{2}\right), \cos \left(2 \pi x^{3}\right)\right)$, $\operatorname{mid}(a, b, c)=a+b+c-\max (a, b, c)-\min (a, b, c)$. В данном случае для всех $\ell$ мы имеем $\mathcal{D}_{M_{0}, \ell}=\mathcal{D}_{f, \ell}$ и все хаотические $H$-сечения оказываются на одном уровне $f=0$. Это является следствием симметрии $f\left(x^{1}+1 / 2, x^{2}+1 / 2, x^{3}+1 / 2\right)=-f(x)$ и неверно в общем случае. Обозначим через $\psi_{1}, \psi_{2}, \psi_{3}$ следующие преобразования проективной плоскости: $\psi_{1}\left(h_{1}: h_{2}: h_{3}\right)=\left(h_{1}: h_{2}+h_{1}: h_{3}+h_{1}\right), \psi_{2}\left(h_{1}: h_{2}: h_{3}\right)=\left(h_{1}+h_{2}: h_{2}:\right.$ $\left.h_{3}+h_{2}\right), \psi_{3}\left(h_{1}: h_{2}: h_{3}\right)=\left(h_{1}+h_{3}: h_{2}+h_{3}: h_{3}\right)$.

ПредлОжениЕ 1. Для указанной выше поверхности $M_{0}=\{f=0\}$ зонами устойчивости являются

$$
\begin{gathered}
\mathcal{D}_{(1: 0: 0)}\left(M_{0}\right)=\left\{\left(h_{1}: h_{2}: h_{3}\right) \in \mathbb{R P}^{2} ; h_{1} \geqslant\left|h_{2}\right|+\left|h_{3}\right|\right\}, \\
\mathcal{D}_{(1: 1: 1)}\left(M_{0}\right)=\left\{\left(h_{1}: h_{2}: h_{3}\right) \in \mathbb{R P}^{2} ; 0 \leqslant h_{1}+h_{2}+h_{3} \leqslant 4 h_{1}, 4 h_{2}, 4 h_{3}\right\}, \\
\mathcal{D}_{\psi_{i_{1}}\left(\psi_{i_{2}}\left(\ldots \psi_{i_{k}}((1: 1: 1)) \ldots\right)\right)}\left(M_{0}\right)=\psi_{i_{1}}\left(\psi_{i_{2}}\left(\ldots \psi_{i_{k}}\left(\mathcal{D}_{(1: 1: 1)}\left(M_{0}\right)\right) \ldots\right)\right),
\end{gathered}
$$

где $\left(i_{1}, \ldots, i_{k}\right)$ - произвольная конечнал последовательность элементов из $\{1,2,3\}$, а также зоны, полученные из перечисленных кубическими симметриями: перестановками и сменами знаков координат.

Таким образом, три зоны $\mathcal{D}_{(1: 0: 0)}, \mathcal{D}_{(0: 1: 0)}, \mathcal{D}_{(0: 0: 1)}$ являются четырехугольниками, а все остальные - треугольниками. Множество $\mathcal{E}(f)$ состоит из четырех частей, замыкания которых гомеоморфны (но не изометричны) треугольнику Серпинского.

Предложение 2. Пересечение $\mathcal{E}(f) \cap\left\{\left(h_{1}: h_{2}: h_{3}\right) \in \mathbb{R P}^{2} ; h_{1}, h_{2}, h_{3}>0\right\}$ cocmoum из всех точек вида $\lim _{k \rightarrow \infty} \psi_{i_{1}}\left(\psi_{i_{2}}\left(\ldots \psi_{i_{k}}((1: 1: 1)) \ldots\right)\right)$, где $\left(i_{1}, i_{2}, \ldots\right)$ - всевозможные бесконечные последовательности элементов множества $\{1,2,3\}$, в которых все три индекса встречаются бесконечное число раз. Остальные точки в $\mathcal{E}(f)$ получаются из указанных кубическими симметриями.

Например, для периодической последовательности $(1,2,3,1,2,3, \ldots)$ мы получаем $\left(1, \alpha^{2}-\alpha, \alpha\right) \in \mathcal{E}(f)$, где $\alpha$ - вещественный корень уравнения $\alpha^{3}-\alpha^{2}-\alpha-1=0$.

ПредЛОЖенИЕ 3. Мера множества $\mathcal{E}(f)$ равна нулю.

Мы оставляем доказательства для более подробной работы. Нам не удалось пока оценить аналитически хаусдорфову размерность множества $\mathcal{E}(f)$ в нашем примере, но несколько независимых приближенных вычислений дают оценку $\approx 1.7$.

Мы благодарим С.П. Новикова за приглашение в университет штата Мэриленд весной этого года, а также сам университет за финансовую поддержку.

\section{Список литературы}

[1] С. П. Новиков, УМН, 37:5 (1982), 3-49. [2] А. В. Зорич, УМН, 39:5 (1984), 235-236. [3] И. А. Дынников, УМH, 54:1 (1999), 21-60. [4] R. De Leo, Experiment. Math., 15:1 (2006), 109-124. [5] S. P. Novikov, A. Ya. Maltsev, J. Statist. Phys., 115:1-2 (2003), 31-46. [6] R. De Leo, SIADS, 2:4 (2003), 517-545. [7] H. S. M. Coxeter, Proc. London Math. Soc., 43 (1937), 33-62.

P. Де Лeo (R. De Leo)

INFN, sez. di Cagliari, Italy

И. А. Дынников (I. A. Dynnikov)

Московский государственный университет

им. М. В. Ломоносова
Представлено С. П. Новиковым Принято редколлегией 20.08.2007 